\title{
WHICH ABELIAN GROUPS CAN SUPPORT A DIRECTED, INTERPOLATION ORDER ?
}

\author{
A. M. W. GLASS
}

\begin{abstract}
We prove that an abelian group can support a directed, interpolation order if and only if it is torsion-free or its quotient by its torsion subgroup is noncyclic. The proof is of an elementary nature. As a consequence of the proof, it is also shown that an abelian group can support a directed, interpolation order if and only if it can support a directed, interpolation, weakly semiisolated order. The paper is completely self-contained so as to be readable by nonspecialists.
\end{abstract}

The study of partially ordered groups started with the theory of totally ordered groups and then lattice-ordered groups. More recently, interpolation groups and directed, interpolation groups have been studied (see e.g., [2]) as a generalisation of lattice-ordered groups.

One natural problem which arises immediately when considering a certain type of partial order is to find a group-theoretic characterisation of those groups that can support the particular kind of partial order under examination. A well-known result of this kind is due to F. W. Levi [4] and is:

An abelian group can support a total order if and only if it is torsion-free.

Since every lattice-ordered group is torsion-free, this condition cannot be weakened when answering the question "Which abelian groups can support a lattice ordering?".

In this paper, an answer will be given to the question posed by the title of the paper; an answer will also be given to similar, related questions which naturally arise here.

The approach taken is very elementary and so sufficient definitions are given in order that the paper can be easily read by all mathematicians.

I. Definitions, notation and basic facts. Let $S$ be a partially ordered set. $S$ is said to be directed if and only if every pair of elements of $S$ has

Received by the editors December 11, 1970.

AMS 1970 subject classifications. Primary 06A60; Secondary 06A55, 20K20, 20K15.

Key words and phrases. Totally ordered group, directed group, interpolation property, weakly semi-isolated group, torsion subgroup of an abelian group, rank of an abelian group, cyclic element of an abelian torsion-free group.

1 This paper represents a portion of the author's Ph.D. thesis at the University of Wisconsin, under the direction of Professor W. Charles Holland, to whom the author wishes to express gratitude.

(c) American Mathematical Society 1972 
an upper and a lower bound in $S$ (i.e., if $s, t \in S$, then there exist $u, v \in S$ such that $u \leqq s, t \leqq v$ ). $S$ is said to be a lattice if and only if every pair of elements of $S$ has a least upper and a greatest lower bound in $S$. $S$ is said to be totally ordered if and only if every pair of elements of $S$ is comparable (i.e., if $s, t \in S$, then $s \leqq t$ or $t \leqq s$ ). The partially ordered set $S$ is said to satisfy the interpolation property if and only if whenever $s, t, u, v \in S$ and $s, t \leqq u, v$, then there exists $x \in S$ (not necessarily unique) such that $s, t \leqq x \leqq u, v$.

Let $\langle G,+\rangle$ be a group (not necessarily abelian). If $G$ is a partially ordered set and the partial order is preserved by addition (i.e., $a, b, g, h \in G$ and $a \leqq b$ implies that $g+a+h \leqq g+b+h$ ), then $G$ is said to be a partially ordered group. Moreover, if the partial order is directed (lattice/total/ satisfies the interpolation property), then $G$ is said to be a directed (lattice/ ordered/interpolation) group. Let $G^{+}=\{x \in G: 0 \leqq x\}$ and $G^{*}=\{x \in G: 0<x\}$. If whenever $x \in G$ and $n$ is a positive integer such that $n x \in G^{+}\left(G^{*}\right) x \in G^{+}\left(G^{*}\right)$, then $G$ is said to be semi-isolated (weakly semi-isolated).

Throughout, $\boldsymbol{Z}(\boldsymbol{R})$ will denote the additive group of integers (reals) and $\boldsymbol{Z}^{+}\left(\boldsymbol{R}^{+}\right)$the positive $(\neq 0)$ integers (reals). $C_{n}$ will denote the cyclic group of order $n$ for each $n \in Z^{+}$and $G \oplus H$ will denote the direct sum of the groups $G$ and $H$. If $X$ is a subset of $G,\langle X\rangle$ will denote the subgroup of $G$ generated by $X$.

The following results are well known and can be found in [1].

If $G$ is a partially ordered group, then $G^{+}$is a normal subsemigroup of $G$ and if $g,-g \in G^{+}$, then $g=0$. If $P$ is a normal subsemigroup of a group $H$ and $h,-h \in P$ iff $h=0$, then $H$ is a partially ordered group under the ordering $h_{1} \leqq h_{2}$ iff $h_{2}-h_{1} \in P$.

If $G$ is a partially ordered group, then $G$ is directed if and only if $\left\langle G^{+}\right\rangle=G$. Indeed, $G$ is directed if and only if every element of $G$ can be expressed as the difference of two elements of $G^{+}$.

II. Results and proofs. The first question to be answered is "Which groups can support a partial order with respect to which they are interpolation groups?".

The answer is every group-for let $G$ be any group with the trivial order defined on it (i.e., $g \leqq h$ iff $g=h$ ); then, vacuously, $G$ is an interpolation group. Actually, what we have shown is:

Every group can support a partial order with respect to which it is a weakly semi-isolated, interpolation group.

For the rest of the paper, only abelian groups will be considered.

Let $G$ be an abelian group and let $T(G)$ be the set of all torsion elements of $G$. It is easy to see that $T(G)$ is a subgroup of $G$ (if we put 0 into $T(G)$ ) and that $G / T(G)$ is torsion-free. Clearly, no element of $T(G)$ can be positive other than 0. (For if $g \in T(G)$ and $g \neq 0$, let $n \in Z^{+}$be such that $n g=0$. 
If $g$ were positive, $0<g<2 g<\cdots<n g=0$, a contradiction.) By Levi's result, it now follows that an abelian group can support a semi-isolated, interpolation order if and only if it is torsion-free.

Also by Levi's result, $H=G / T(G)$ can support a total order, say $\leqq_{H}$. Let $G^{+}=\left\{g \in G: g=0\right.$ or $\left.g+T(G)_{H}>T(G)\right\}$. It is easy to see that $G$ is a partially ordered group with respect to the ordering defined by $G^{+}$. In fact, if $H \neq\{0\}, G$ is directed with respect to this partial ordering. Consequently,

THEOREM (ŠIMBIREVA). An abelian group can support a directed partial ordering if and only if it is the trivial group, or its quotient by its torsion subgroup is not the trivial group.

From the above remarks, it might be supposed that if an abelian group is to support a directed, interpolation order, it would be enough to satisfy the conditions of Šmbireva's theorem. That this might not be the case is seen by letting $G=\boldsymbol{Z} \oplus C_{2}$ ordered by: $(m, n x)>0$ iff $m>0 . G$ is a directed group which is not an interpolation group as $(1, x),(1,0)>$ $(0, x),(0,0)$, but there does not exist $g \in G$ such that

$$
(1, x),(1,0) \geqq g \geqq(0, x),(0,0) .
$$

The question "Which abelian groups can support a directed, interpolation order?" together with the easier and more intuitive problem "Which abelian groups can support a directed, interpolation, weakly semi-isolated order?" are simultaneously attacked.

The next series of lemmas will help to characterise both classes of groups.

LEMMA 1. If $G$ is an abelian, weakly semi-isolated group, then for each $g \in T(G)$ and $y \in G^{*},-y<g<y$.

Proof. If $n g=0$ for some $n \in Z^{+}$and $g<x$, then $0<x-g$. Hence $0<n(x-g)=n x-n g=n x$. Therefore $0<x$. If $y \in G^{*}$, then $g<y+g$; so $0<y+g$. Similarly, $0<y-g$. Thus $-y<g<y$.

Let $H=G / T(G)$ and $H^{+}=\{h \in H: h=T(G)+g$ for some $g \geqq 0\}$. Then $H$ is a partially ordered group. $H$ is semi-isolated if $G$ is weakly semi-isolated.

COROLLARY 1.1. If $G$ is an abelian, weakly semi-isolated group, then any two elements in the same coset of $T(G)$ have the same upper and lower bounds in $G$.

In view of Lemma 1, we now examine $H$.

LEMMA 2. For each $h \in H \backslash\{0\}, Q(h)=\{g \in H: m h=n g$ for some $m \in Z$ and some $\left.n \in Z^{+}\right\}$is a subgroup of $H$ and $H=\bigcup\{Q(h): h \in H \backslash\{0\}\}$. Furthermore, $Q(h)=Q(k)$ or $Q(h) \cap Q(k)=\{0\}$ if $k \in H \backslash\{0\}$. 
Let $h \in H \backslash\{0\}$. $h$ is said to be cyclic if and only if $Q(h)$ is a cyclic subgroup of $H$.

LEMMA 3. If $\leqq$ is a partial order on $H$ with respect to which $H$ is semiisolated, then for each $h \in H \backslash\{0\}, Q(h)$ is either trivially ordered or an ordered group.

Let $A$ be an indexing set for $\{Q(h): h \in H \backslash\{0\}\} .|A|$, the cardinality of $A$, is called the rank of $H$ (see [3]). From the earlier example, if $H$ is of finite rank, then it may not be possible to make $G$ a directed, interpolation, weakly semi-isolated group.

LEMMA 4. If $h_{1}, h_{2} \in H \backslash\{0\}, Q\left(h_{1}\right) \neq Q\left(h_{2}\right)$ and $m, n, r$ and $s$ are integers such that $(m, n),(r, s) \neq(0,0)$, then $Q\left(m h_{1}+n h_{2}\right)=Q\left(r h_{1}+s h_{2}\right)$ if and only if $n=s=0$ or $n$ and $s$ are both different from 0 and $m / n=r / s$. Hence $H$ is of rank 1 or of infinite rank.

The following lemma completes the examination of the case that $H$ is of finite rank.

LEMMA 5. If $H$ has rank 1 and $T(G) \neq\{0\}$, then $G$ can support a directed, interpolation, weakly semi-isolated order if and only if every $h \in H \backslash\{0\}$ is noncyclic, where $H=G / T(G)$.

Proof. If $G$ can support a weakly semi-isolated, directed, interpolation order, then $H \neq\{0\}$. Let $\leqq$ be the partial order on $G$ and $\leqq H$ the induced partial order on $H$. Let $g \in T(G) \backslash\{0\}$. Since $G$ is directed, there exists $x \in G$ such that $x \geqq g, 0$ and $x \notin T(G)$ because $T(G)$ is trivially ordered. Let $X=x+T(G)_{H}>T(G)$. By Lemma 3, $Q(X)$ is an ordered group. By way of contradiction, assume $Q(X)$ is cyclic. Let $Y$ be a generator of $Q(X)$ and choose $y \in G$ such that $Y=y+T(G)$. Without loss of generality, $Y$ can be chosen to be positive with respect to $<_{H}$. Thus there exists $k \in T(G)$ such that $y+k>0$. By Corollary $1.1, y>0, g$; also $y+g>0, g$. Since $G$ is an interpolation group, there exists $f \in G$ such that $y, y+g \geqq f \geqq g, 0$. Hence $Y \geqq F \geqq 0$ where $F=f+T(G)$. But $f \geqq 0, g$ and $T(G)$ is trivially ordered; thus $f \notin T(G)$; so $F \neq 0$. Now $F \in Q(Y)$ and $Y$ is a cyclic generator of $Q(Y)$. Therefore, $F=Y$. Consequently, there exists $k^{\prime} \in T(G)$ such that $y=f+k^{\prime}$. But $y \geqq f$, so $f+k^{\prime} \geqq f$. Hence $k^{\prime}=0$ and $y=f$. However, $y+g \geqq f$. It follows that $g \geqq 0$. As a result, $T(G)$ is not trivially ordered, a contradiction. As $|A|=1, Q(h)=Q(X)$ for all $h \in H \backslash\{0\}$. Therefore, every $h \in H \backslash\{0\}$ is noncyclic.

Conversely, if $Q(h)$ is not cyclic, define $G^{+}=\{g \in G: g=0$ or $T(G)<$ $T(G)+g\}$ where $\leqq$ is any order on $H=Q(h)$ which makes $H$ an ordered group (the existence of such an ordering is guaranteed by Levi's theorem). It is easy to check that the partial ordering given by $G^{+}$makes $G$ a directed, 
interpolation, weakly semi-isolated group (the interpolation property follows from Corollary 1.1 and the fact that the total order on $H$ is dense (if $x<y$, then there exists $z$ such that $x<z<y$ ) by hypothesis).

A well-known fact is:

LEMMA 6. If $M$ is a subgroup of $\boldsymbol{R}$ and $\boldsymbol{R}$ has its usual ordering, then $M$ is either cyclic or dense in $\boldsymbol{R}$.

With the above lemmas, it is now a relatively simple matter to characterise the class of abelian groups which can support a directed, interpolation order. This is done in the following theorem:

THEOREM A. An abelian group $G$ can support a directed, interpolation order if and only if $G$ is torsion-free or $G / T(G)$ is noncyclic.

Proof. If $G$ is torsion-free, then the result follows from Levi's theorem; so assume that $T(G) \neq\{0\}$. Suppose $G / T(G)$ is noncyclic. Either there exists $h \in H=G / T(G)$ such. that $h$ is noncyclic (and $\neq 0$ ), or there exist cyclic $h_{1}, h_{2} \in H \backslash\{0\}$ such that $Q\left(h_{1}\right) \neq Q\left(h_{2}\right)$. If there exists $h \in H$ such that $h$ is noncyclic, then, as $H / Q(h)$ is torsion-free, it can support a total order, $\leqq_{K}$, say, where $K=H / Q(h)$. Also $Q(h)$ can be ordered by $\leqq_{h}$, say, so as to be an ordered group. Now define $G^{+}=\{g \in G: g=0$ or $\bar{g}=g+T(G) \in Q(h)$ and $T(G)<_{h} \bar{g}$ or $\bar{g} \notin Q(h)$ and $\left.\bar{g}+Q(h)_{K}>Q(h)\right\}$. It is immediate that with this partial ordering, $G$ is a directed, interpolation, weakly semiisolated group by the argument used at the end of the proof of Lemma 5. If there exist $h_{1}, h_{2} \in H \backslash\{0\}$, cyclic, such that $Q\left(h_{1}\right) \neq Q\left(h_{2}\right)$, let

$$
L=\bigcup\left\{Q\left(m h_{1}+n h_{2}\right): m, n \in Z, \operatorname{not} m=n=0\right\} .
$$

Let $K=H / L . K$ is torsion-free and so can support a total order, $\leqq_{K}$, say. Let $e$ have its usual number-theoretic meaning. Define $m h_{1}+n h_{2}>0$ iff $m e+n>0$, and if $x \in Q\left(m h_{1}+n h_{2}\right)$, there exist $p \in Z$ and $q \in Z^{+}$such that $q x=p\left(m h_{1}+n h_{2}\right)$. Then $0<_{L} x$ iff $0<_{L} p m h_{1}+p n h_{2} . L$ is an ordered group under $\leqq_{L}$ and is order-isomorphic to a dense subgroup of the totally ordered reals, by Lemma 6. Define $G^{+}=\{g \in G: g=0$ or $\bar{g}=g+$ $T(G) \in L$ and $T(G)<_{L} \bar{g}$ or $\bar{g} \notin L$ and $\left.L<_{K} \bar{g}+L\right\}$. Again with this partial ordering, by the same reasoning, it is exhibited that $G$ is a directed, interpolation, weakly semi-isolated group.

It remains only to prove that if $G$ can support a directed, interpolation order and $T(G) \neq\{0\}$, then $G / T(G)$ is noncyclic. By way of contradiction, suppose that $G$ can support such an order, $T(G) \neq\{0\}$ and $G / T(G)=H=$ $\langle h\rangle$, say. Let $\leqq$ be the partial order on $G$ and $\leqq_{H}$ the partial order induced on $H$. Since $\left(H, \leqq_{H}\right)$ is the image under an $o$-homomorphism (a group homomorphism such that if $a \leqq b$, then the image of $a$ is less than the image of $b$ ) of the directed group $G$, it is also directed. Hence it is not 
trivially ordered and without loss of generality, some positive multiple of $h$ is positive in $\left(H, \leqq_{H}\right)$. Let $k \in T(G) \mid\{0\}$ and let $n$ be the order of $k$. Since $G$ is directed, there exists $g \in G$ such that $g \geqq k, 0 . g+T(G)=m h$ for some $m \in Z^{+}(T(G)$ is trivially ordered so $m \neq 0)$. Choose $g$ so that $m$ is minimal. Now $(n-1) g+k=(n-1) g-(n-1) k=(n-1)(g-k) \geqq 0$. Hence $(n-1) g+k$, $g \geqq k$, 0 ; so there exists $x \in G$ such that $(n-1) g+k, g \geqq x \geqq k, 0$. Thus $T(G)+g_{H} \geqq T(G)+x_{H} \geqq T(G)$, and $x \notin T(G)$ because $T(G)$ is trivially ordered. By the "minimality" of $g$ and the fact that $g \geqq x, T(G)+x=$ $T(G)+g$, and so $g=x$. It follows that $(n-1) g+k \geqq g$. Therefore $(n-2) g+k$, $g \geqq k, 0$. Continuing in this way, we obtain $g+k, g \geqq k, 0$. Consequently, there exists $x \in G$ such that $g+k, g \geqq x \geqq k$, 0 . As before, $g=x$, whence $g+k \geqq g$. Thus $k \geqq 0$, a contradiction to the fact that $T(G)$ is trivially ordered. Hence the proof is complete.

The example $G=Z \oplus C_{2}$ is now seen to be in some sense crucial, and is the intuitive key to the solution of the problem. Thus it is truly the enfant terrible of the piece.

As a corollary of the proof, we obtain:

THEOREM B. Any abelian directed, interpolation group can be reordered so as to be a directed, interpolation, weakly semi-isolated group.

\section{REFERENCES}

1. L. Fuchs, Partially ordered algebraic systems, Pergamon Press, New York; Addison-Wesley, Reading, Mass., 1963. MR 30 \#2090.

2. ——, Riesz groups, Ann. Scuola Norm. Sup. Pisa (3) 19 (1965), 1-34. MR 31 \#4843.

3. A. G. Kuroš, Theory of groups, 2nd ed., GITTL, Moscow, 1953; English transl., Chelsea, New York, 1956. MR 15, 501; 18, 188.

4. F. W. Levi, Arithmetische Gesetz im Gebiete diskreter Gruppen, Rend. Circ. Mat Palermo 35 (1913), 225-236.

Department of Mathematics, University of Wisconsin, Madison, Wisconsin 53706

Current address: Department of Mathematics, Bowling Green State University, Bowling Green, Ohio 43403 